\title{
Failure Modes in Surface Micromachined MicroElectroMechanical Actuators
}

\author{
S. L. Miller, M. S. Rodgers, G. LaVigne, J. J. Sniegowski, P. Clews, D. M. Tanner, K. A. Peterson \\ Sandia National Laboratories \\ Mail Stop 1080 \\ P.O. Box 5800 \\ Albuquerque, NM 87185-1080 \\ http://www.mdl.sandia.gov/Micromachine

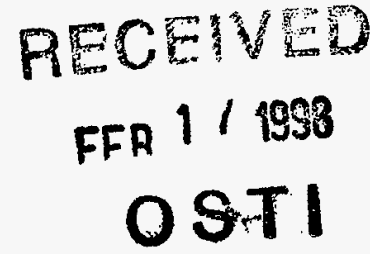

\begin{abstract}
In order for the rapidly emerging field of MicroElectroMechanical Systems (MEMS) to meet its extraordinary expectations regarding commercial impact, issues pertaining to how they fail must be understood. We identify failure modes common to a broad range of MEMS actuators, including adhesion (stiction) and friction-induced failures caused by improper operational methods, mechanical instabilities, and electrical instabilities. Demonstrated methods to mitigate these failure modes include implementing optimized designs, model-based operational methods, and chemical surface treatments.
\end{abstract}

Keywords: microengine, failure mechanisms, MEMS failure modes

\section{Introduction}

While anticipated by many to be a revolutionary and enabling new technology, the issues pertaining to how MicroElectroMechanical Systems (MEMS) fail are not well understood. The current situation is only natural for a technology that is still in its infancy, similar to the state of integrated circuit technology 30 years ago. The primary advances in MEMS have been focussed on technology development, i.e. creating new ways to fabricate prototype devices. The objective has typically been to demonstrate functionality of prototypes, hence demonstrating the utility and potential benefit of a given technology. The somewhat less attractive task of understanding failure modes and mechanisms has been left largely unaddressed, at least in the public literature.

In spite of the lack of a significant body of publicly available reliability and failure information, several companies have successfully commercialized MEMS products. For example, Analog Devices has commercialized a family of micromachined accelerometers, many of which comprise automobile airbag trigger systems. [1] Texas Instruments has commercialized digital mirror display systems, at the heart of which is an array of microscopic micromachined mirrors. [2] Hewlett Packard's extremely successful ink jet print heads are micromachined devices. [3] To be successful, it is anticipated that these and other companies producing MEMS products have had to mount significant internal performance enhancement and reliability assessment programs, the details of which would obviously be proprietary.

In this paper we discuss the types of reliability issues identified and being addressed by Sandia National Laboratories, with a twofold objective. One obvious objective is to allow other MEMS researchers to benefit from the insight gained from our rather significant internal MEMS program. The other objective is to inform reliability researchers not familiar with MEMS of some very challenging opportunities that must be addressed to further advance the growing MEMS revolution.

\section{Background}

\section{Fabrication Technology}

The primary MEMS technologies developed at Sandia are in the category of surface micromachining. [4] Our SUMMiT fabrication process [5] involves film deposition, patterning, and etching, with this sequence being repeated over and over to create the resulting

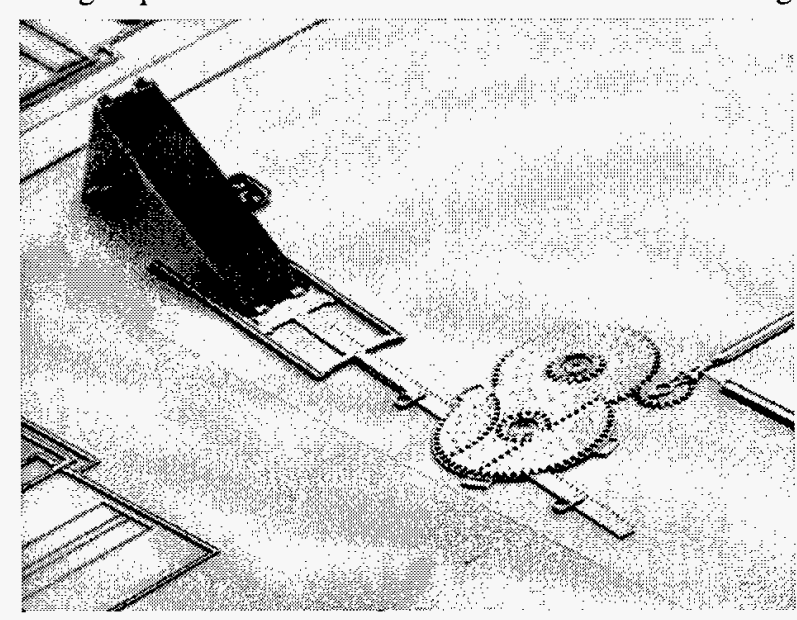

Fig. 1. The microengine pinion gear (right) powers a torque convertor, consisting of a series of multi-level rotating gears. The torque convertor actuates an out-of-plane mirror by pushing a linear rack. The self assembled system is batch fabricated. 


\section{DISCLAIMER}

This report was prepared as an account of work sponsored by an agency of the United States Government. Neither the United States Government nor any agency thereof, nor any of their employees, makes any warranty, express or implied, or assumes any legal liability or responsibility for the accuracy, completeness, or usefulness of any information, apparatus, product, or process disclosed, or represents that its use would not infringe privately owned rights. Reference herein to any specific commercial product, process, or service by trade name, trademark, manufacturer, or otherwise does not necessarily constitute or imply its endorsement, recommendation, or favoring by the United States Government or any agency thereof. The views and opinions of authors expressed herein do not necessarily state or reflect those of the United States Government or any agency thereof. 
devices. The primary materials used for the layers are polycrystalline silicon (often simply termed poly), and silicon dioxide. Silicon nitride is also occasionally used. At the end of the fabrication process, the silicon dioxide is removed by a wet chemical etch, resulting in the mechanical poly layers being free to move. ChemicalMechanical Polishing (CMP) is also used in the fabrication process to permit greater design flexibility. [6]

The pop-up mirror shown in Fig. 1 illustrates one of the key advantages of the surface micromachine SUMMiT fabrication process: the devices are completely batch fabricated on a silicon wafer using tools common to the IC manufacturing industry, and hence are inexpensive to make. The mirror system is fabricated completely assembled; no piece part assembly is required. Specifically, the electrostatic comb drives, torque conversion gears, linear rack, and mirror hinges are completely assembled and functional upon completion of the final release etch.

\section{Mechanical properties}

Polysilicon is the primary mechanical material comprising surface micromachined MEMS. It is ideal for this purpose since it is strong, hard, easy to deposit and etch (compatible with IC processing), and can be made relatively stress free. The devices we have made thus far (including mechanical locking systems, optical shutters, positionable mirrors, and torque conversion systems $[7,8])$ are observed to be quite robust. Support springs do not break, pin joints do not typically break, gear hubs and gear teeth do not break, hinges do not break, alignment guides and clips do not break, and flex joints do not break. [9] The robustness of our initial designs, which did not make extensive use of mechanical modeling, make it evident that, at least presently, materials strength is not a key limiting factor in MEMS performance and reliability. If mechanical robustness (e.g. fracture strength) is not a primary reliability issue with MEMS, then what is?

\section{Surface properties}

The relative magnitude of forces impacting the dynamics and performance of conventional macroscopic systems is quite different than for MEMS. At the size scale of MEMS (microns), gravitational forces become negligible. By far the most dominant forces at the microscale are associated with contacting or rubbing surfaces. Adhesion of contacting surfaces (often termed stiction) and friction between sliding surfaces have the greatest impact on MEMS performance and reliability. There are several root causes that give rise to adhesion and friction-related failures.

\section{Failure Modes: Root Causes}

\section{Capillary forces}

The final step in the process of fabricating surface micromachined MEMS is a wet chemical etch, which removes the silicon dioxide matrix that encapsulates the moveable mechanical structures. Removal of the wafer from the liquid etchant results in a meniscus (liquid-air interface) that often pulls moveable structures into contact via capillary forces. Once in contact, and even after drying, the surfaces often remain in contact due to various types of adhesion forces (e.g. capillary, van Der Walls, electrostatic due to trapped charge). To illustrate, in Fig. 2(a) a folded spring structure is adhered to the substrate after drying, in contrast with the desired free configuration shown in Fig. 2(b). Meniscus-induced contact can be eliminated using drying techniques such as freeze sublimation [10] or super-critical drying, [11]

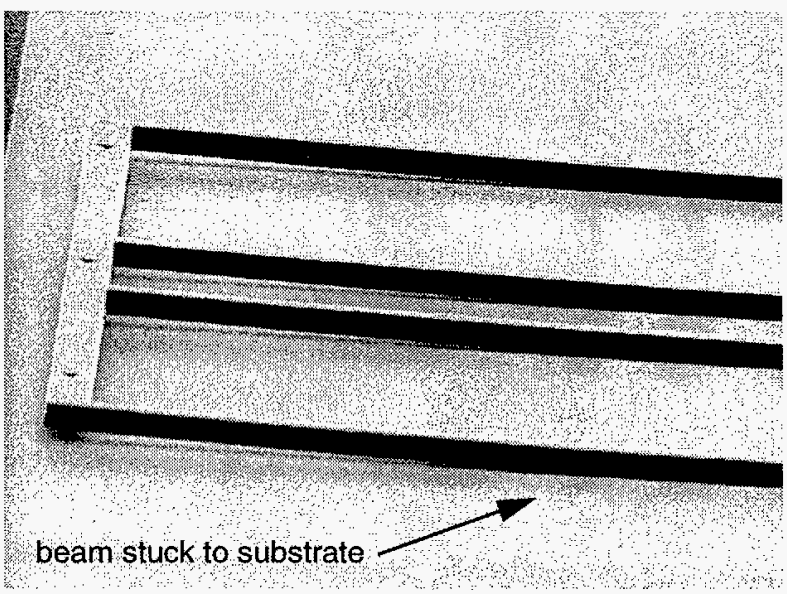

(a)

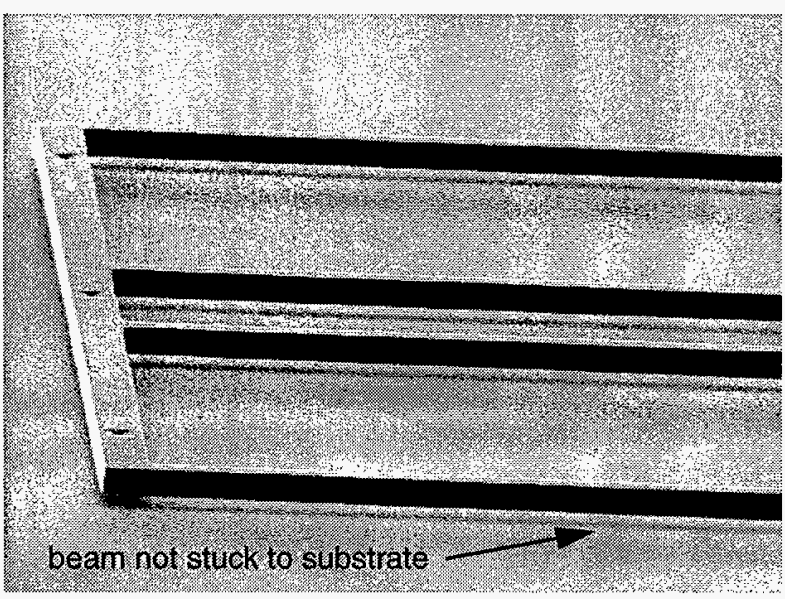

(b)

Fig. 2. A comb drive support spring is adhered to the substrate, resulting in failure. Treatment with chemicals to make surfaces hydrophobic during the drying process results in free support beams. 


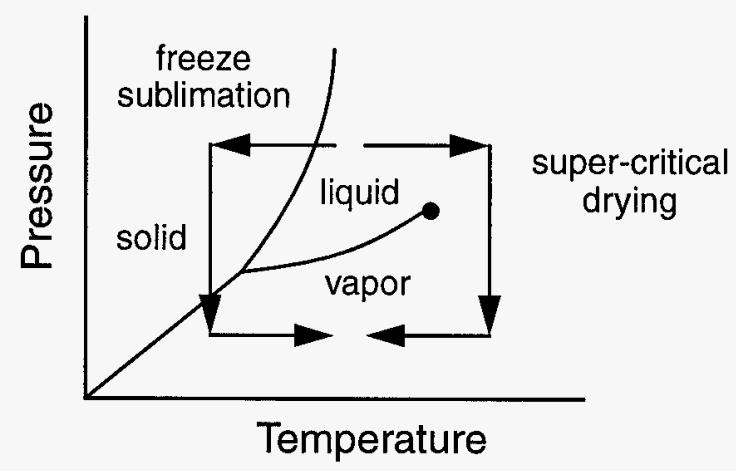

Fig. 3. Phase diagram illustrating two paths (super-critical drying and freeze sublimation) to pass from the liquid to vapor state without encountering a liquid-vapor interface. The occurrence of a liquid-vapor interface can lead to adhesion between surfaces brought into contact by capillary forces.

whose basic principles are illustrated by the phase diagram in Fig. 3. The basic idea is to avoid the formation of the meniscus by taking one of two paths in the phase diagram. Attractive capillary forces can also be mitigated by coating potentially contacting surfaces with hydrophobic chemicals, such as coupling agents like ODTS. [12] Developing manufacturable methods to achieve successful drying continues to be an active area of research.

Even if MEMS are successfully dried using one or more of the above methods, surfaces brought into contact after successful drying can still adhere, resulting in impaired functionality or failure. Moreover, when microscopic surfaces are exposed to such uncontrolled environments as air with changing humidity or packages with epoxy vapors, their properties can change significantly (typically for the worse). While coupling agents and other coating materials are being investigated to reduce stiction, the long term effectiveness remains largely unexplored. The development of manufacturable methods to stabilize the properties of surfaces is critical to the continued commercialization of MEMS products.

Stiction can in many cases be mitigated by clever design modifications. The idea is to implement designs that stiffen the structures in the direction of motion where stiction is most likely to occur. For example, the support springs exhibiting stiction in Fig. 2(a) can be made stiffer by the inclusion of another mechanical layer of polysilicon, as shown in Fig. 4. Tripling the thickness of the beams by the inclusion of another layer effectively increases the stiffness by a factor of nearly 30. Such springs rarely adhere to the substrate. Combining coupling agent treatments with this type of design-related vertical stiffening has improved yield of comb drive structures from virtually zero to nearly 100 percent.

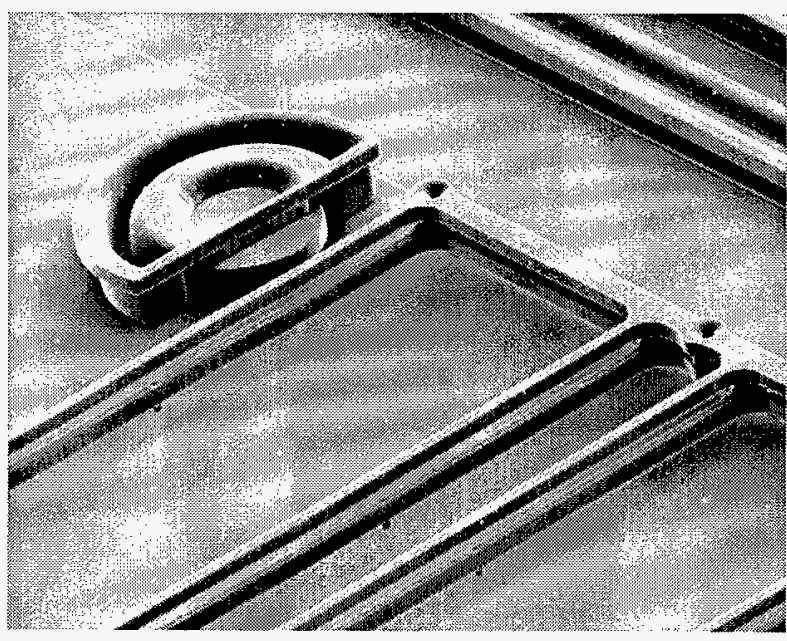

Fig. 4. Stiffening the support springs by the addition of another level of polysilicon effectively mitigates adhesion to the substrate as a failure mode for this structure.

\section{Operational Methods}

Many implementations of MEMS actuators contain physically constrained systems, such as the microengine shown in Fig. 5, for example. The motion of the gear is constrained by the hub on which it rotates, and the comb drives are constrained by the linkages connecting them to the pinion gear. All the forces associated with the system balance to result in the dynamical behavior of the system. Control is provided by the actuating electrical drive signals. If appropriate drive signals are not provided to the comb actuators,

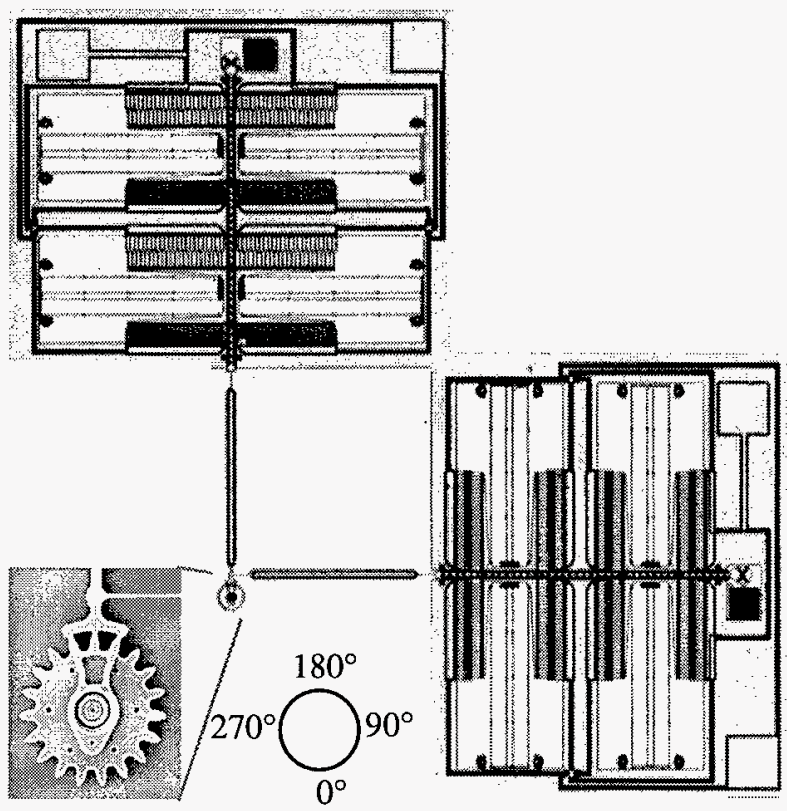

Fig. 5. The microengine consists of orthogonally oriented linear comb drive actuators mechanically connected to a rotating gear capable of providing torque to a load device. The as-fabricated, neutral-spring position of the pinon gear is $0^{\circ}$. 
significant constraint forces can result to compensate for other forces, such as inertia. Constraint forces, particularly of the type associated with rubbing surfaces, are observed to result in premature failure. It is thus desirable to operate MEMS in a way that minimizes forces between rubbing surfaces.

To consider a macroscopic analogy, consider "popping the clutch" of an automobile with a standard transmission. The abrupt force resulting from the inertia of the flywheel acting on the transmission results in excessive forces very detrimental to the entire drive train. The clutch was designed to enable the system to operate with reduced parasitic forces, significantly increasing reliability and lifetime. In exactly the same way, the methods by which MEMS are operated can significantly impact their reliability and lifetime.

Continuing with the microengine as an example regarding operational methods, we examine the starting and stopping process. The model relevant to the starting and stopping process is based on the schematic of the microengine shown in Fig. 6, and follows the derivation given in [13]. The force between the gear and the linkage mechanism is resolved into radial and tangential components $F_{r}$ and $F_{l}$, respectively. The electrostatic, restoring spring, and viscous damping forces in the $y$ direction are expressed as:

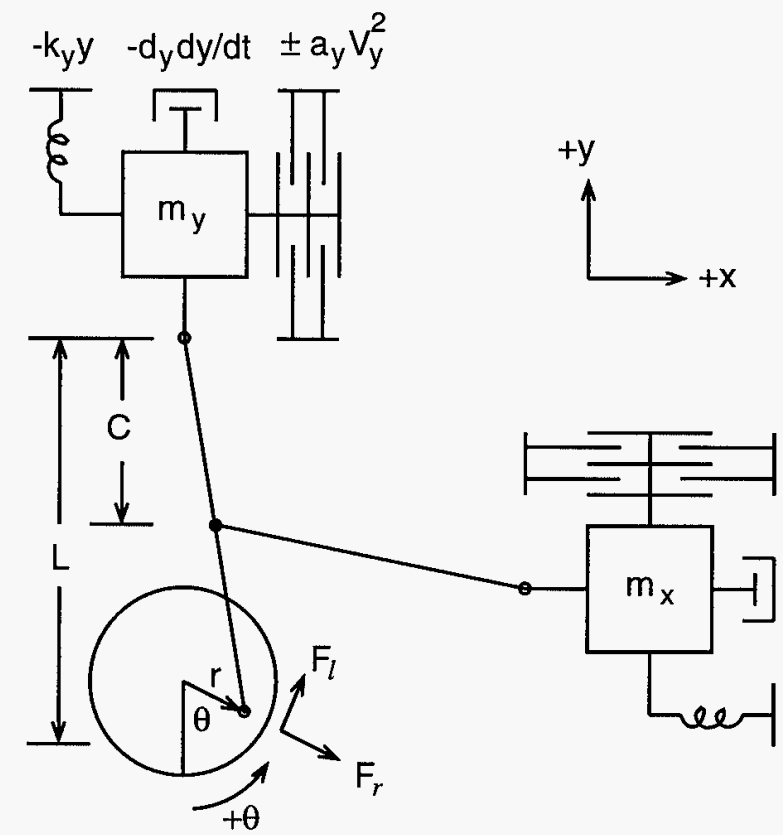

Fig. 6. Forces acting at the pinion gear pin joint $\left(F_{r}\right.$ and $\left.F_{l}\right)$ result from inertial forces, spring forces, damping forces, electrostatic actuation forces, and frictional forces.

$$
\begin{gathered}
F_{y}= \pm a_{y} V_{y}^{2} \\
F_{y}=-k_{y} y \\
F_{y}=-d_{y} \frac{d y}{d t} .
\end{gathered}
$$

where $a_{y}$ is the electrostatic force constant, $k_{y}$ is the restoring spring force constant, and $d_{y}$ is the coefficient of viscous damping. Similar forces exist in the $x$ direction. To simplify the form of the resulting equations, we define the following terms:

$$
\begin{aligned}
\delta_{x} & =\frac{d_{x}}{2 m_{x}} \\
\omega_{x} & =\sqrt{\frac{k_{x}}{m_{x}}} \\
\gamma & =\frac{C}{L},
\end{aligned}
$$

where $m_{x}$ is the mass of the structure moving in the $x$ direction, $\delta_{x}$ is the viscous damping ratio, $\omega_{x}$ the resonant frequency of the moving mass, and $C$ and $L$ are geometrical quantities illustrated in Fig. 6. Expressions similar to Eqs. (4) and (5) also exist for the $y$ direction of motion. The effects of the small angle deflections of the linkages are relatively insignificant, and hence are neglected. Omitting the details of the derivation, we solve Newton's equation $\Sigma F=m a$ for both $m_{x}$ and $m_{y}$, and obtain

$$
\begin{aligned}
\frac{F_{r}}{k r}= & \gamma \frac{ \pm a_{x}}{k r} V_{x}^{2} \sin (\theta)-\frac{ \pm a_{y}}{k r} V_{y}^{2} \cos (\theta)+\cos (\theta)-1 \\
& +\sin (\theta) \cos (\theta)\left[\ddot{\theta}\left(\frac{1}{\omega_{y}^{2}}-\frac{\gamma^{2}}{\omega_{x}^{2}}\right)+2 \dot{\theta}\left(\frac{\delta_{y}}{\omega_{y}^{2}}-\frac{\gamma^{2} \delta_{x}}{\omega_{x}^{2}}\right)\right] \\
& +\dot{\theta}^{2}\left[\frac{\cos ^{2}(\theta)}{\omega_{y}^{2}}+\frac{\gamma^{2} \sin ^{2}(\theta)}{\omega_{x}^{2}}\right]+\left(1-\gamma^{2}\right) \sin ^{2}(\theta)
\end{aligned}
$$

and

$$
\begin{aligned}
\frac{F_{l}}{k r}= & \gamma \frac{ \pm a_{x}}{k r} V_{x}^{2} \cos (\theta)+\frac{ \pm a_{y}}{k r} V_{y}^{2} \sin (\theta)-\sin (\theta) \\
& -\dot{\theta}^{2} \sin (\theta) \cos (\theta)\left(\frac{1}{\omega_{y}^{2}}-\frac{\gamma^{2}}{\omega_{x}^{2}}\right)-\frac{\gamma^{2} \cos ^{2}(\theta)}{\omega_{x}^{2}}\left(\ddot{\theta}+2 \delta_{x} \dot{\theta}\right) \\
& -\frac{\sin ^{2}(\theta)}{\omega_{y}^{2}}\left(\ddot{\theta}+2 \delta_{y} \dot{\theta}\right)+\left(1-\gamma^{2}\right) \sin (\theta) \cos (\theta)
\end{aligned}
$$

where $F_{r}$ and $F_{l}$ are the radial and load (tangential) components of the force acting on the gear by the drive arm (see fig. 6). Because of the design symmetry, in this 
derivation we let $k=k_{x}=k_{y}$. Note also that the forces are divided by the convenient scale factor $k r$, which is the force whose magnitude is equal to that exerted by the springs when the comb drive is displaced a distance of one radius. Finally note the derivation of Eqs. (7) and (8) makes no specific assumptions regarding the drive voltages $V_{x}$ and $V_{y}$.

Consider the case where the engine is being operated using model-based drive signals $V_{x}(\mathrm{t})$ and $V_{y}(\mathrm{t})$ [14] to minimize the constraint force $F_{r}$, and hence minimize the force between the gear and the hub. Suppose the drive voltages are then abruptly dropped to zero to stop the engine. At that same instant, the electrostatic force that was being supplied to the comb drive is now provided by the pin joint $\left(F_{r}\right.$ and $F_{l}$ of Eqs. (7) and (8)), and ultimately by the interface between the gear and the hub on which it is rotating. These constraint forces can easily experience momentary increases of well over an order of magnitude, resulting in significant wear and premature failure.

To demonstrate how constraint forces can result in failure, identical microengines were operated using either model-based drive signals designed to minimize $F_{r}$, or simple square wave drive signals. The microengines operated using square wave drive signals typically failed after on the order of $10 \mathrm{star} / \mathrm{stop}$ cycles. Failure is evidenced by a change in the rubbing surfaces between the gear and hub that increases the frictional drag force to the point that the gear is no longer able to rotate on the hub. In contrast, microengines operated using model-based drive signals have demonstrated millions of start/stop cycles, a five order of magnitude increase in stop/start endurance.

So summarize, improper operational methods, i.e. those not using model-based drive signals designed to minimize parasitic constraint forces, can significantly degrade performance of MEMS. Consequently, the method of operation must be seriously considered when developing MEMS that are to be highly reliable.

\section{Mechanical Instabilities}

Microelectromechanical systems are just that: micromechanical devices typically actuated by electrical forces. Electrical forces such as capacitive attraction can depend sensitively on the geometry of the attracting electrodes, and must be carefully considered when implementing constraint methods for moving mechanical elements. Otherwise, undesired forces can result in mechanical instabilities causing both performance degradation and premature failure. We illustrate this with a case history involving the comb drive, a basic actuation element that is in widespread

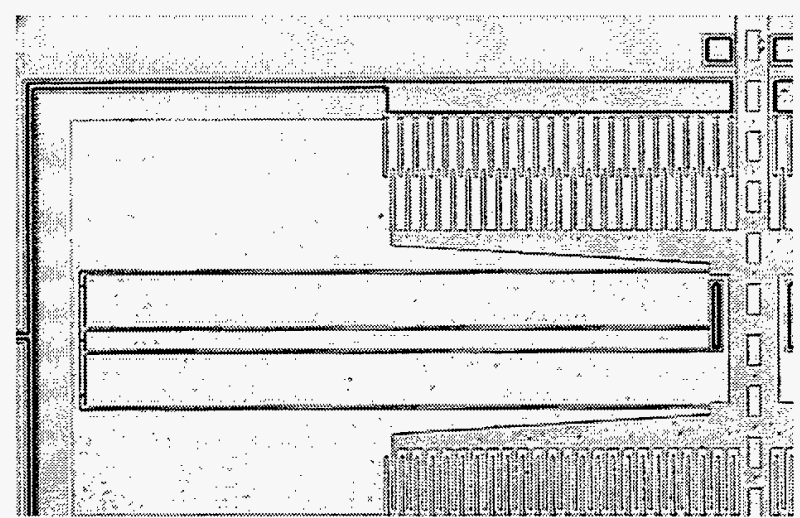

(a)

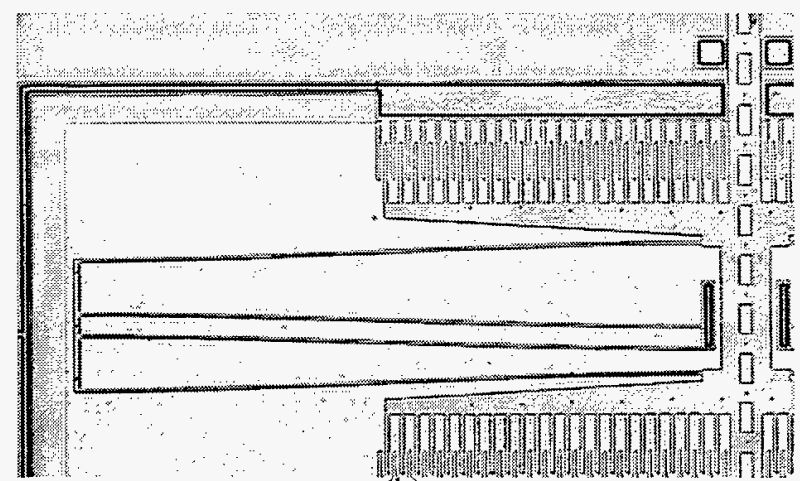

(b)

Fig. 7. The electrostatically actuated comb drive is supported by folded springs to provide lateral and torsional stiffness as the comb shuttle moves from the equilibrium position (a) to the fully displaced position (b). Only the left half of the symmetric comb drive is shown.

use. [15]

The elements of a comb drive actuator are shown in Fig. 7 (the same type of actuator powering the microengine in Fig. 5). A voltage applied between the interdigitated comb fingers results in an attractive force. The moveable comb shuttle is supported by a set of folded springs designed to provide lateral and torsional stiffness. The intent of the design is to limit the shuttle to the desired one-dimensional motion. Unfortunately, a mechanical instability can occur which results in degraded performance and several types of failure.

A mechanical instability occurs because, in addition to the electrostatic force acting on the shuttle in the desired direction of motion, relatively large electrostatic forces are acting on the comb fingers in the lateral direction. These lateral forces cancel when the shuttle is perfectly aligned, but become very asymmetric with only slight perturbations in the lateral direction. The functional form of the net lateral force is illustrated in Fig. 8. The lateral stiffness of the support springs typically keep the comb drive properly aligned in a stable configuration. When the shuttle is actuated and 


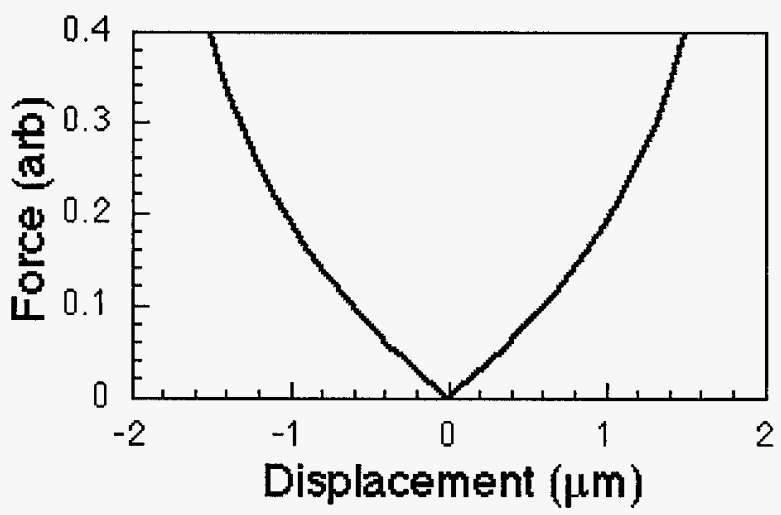

Fig. 8. The rapid increase of the lateral force with lateral comb displacement clearly indicates the need to keep the comb shuttle properly centered. Displacements of only a few tenths of a micron result in a significantly unbalanced lateral force.

the support springs flex, their lateral stiffness decreases with increasing comb drive displacement. The existence of slight off axis torques or vibrations, particularly when the comb drive is at its maximum displacement, can cause the support springs to buckle in the lateral direction, as shown in Fig. 9. When this happens, the lateral electrostatic force clamps the comb drive against one or more alignment guides, where friction then causes its motion to cease until the applied voltage drops below the critical unbuckling value.

This so-called lateral clamping is evident in the motion of a microengine (see Fig. 5) which is actuated by two orthogonally positioned comb drives. The angular position of the pinion gear, measured during high speed operation, is shown in Fig. 10. Note that near the $180^{\circ}$ position (the maximum displacement of the comb drive) the motion of the gear abruptly ceases. Careful stroboscopic analysis during operation clearly indicates this cessation of motion is directly caused by lateral clamping of the comb drive actuator.

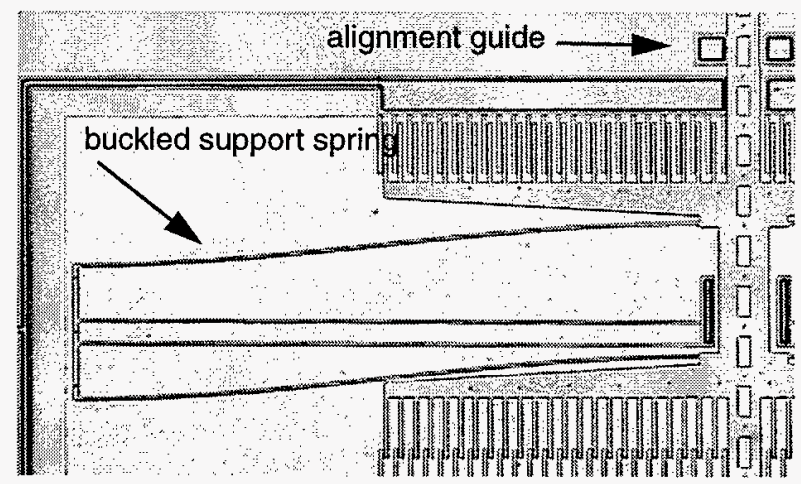

Fig. 9. When fully displaced, the comb drive support springs can buckle, allowing the comb drive to clamp laterally against one of the alignment guides.

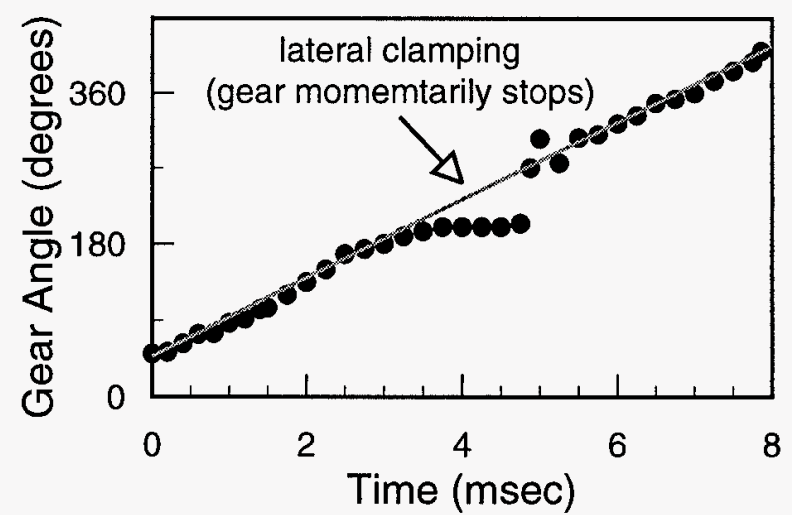

Fig. 10. Lateral clamping of a comb drive actuating the microengine results in fluctuations in the instantaneous angular speed of the pinion gear, leading to failure.

In addition to the degradation in performance (the time-dependent position of the output gear deviates from the desired path), lateral clamping directly results in two distinct types of failures. First, the abrupt angular acceleration experienced by the gear during each revolution results in severe parasitic frictional forces between the gear and the hub on which it rotates, as well as in the pin joint connecting the gear to the linkage arm. The excessive force between the rubbing surfaces causes the gear to bind on the hub, and rotation to cease. The second failure mode occurs when an individual comb drive finger deflects sufficiently to contact an adjacent finger. When this occurs, the two typically fuse together, causing immediate and abrupt failure of the comb drive.

Fortunately, lateral clamping can be mitigated by design modifications, such as that shown in Fig. 11. By changing the shape of the interface between the alignment guides and the comb shuttle, the gap required during the fabrication process can be maintained, but is reduced when the shuttle is actuated. By physically limiting the lateral displacement, the lateral forces (Fig.

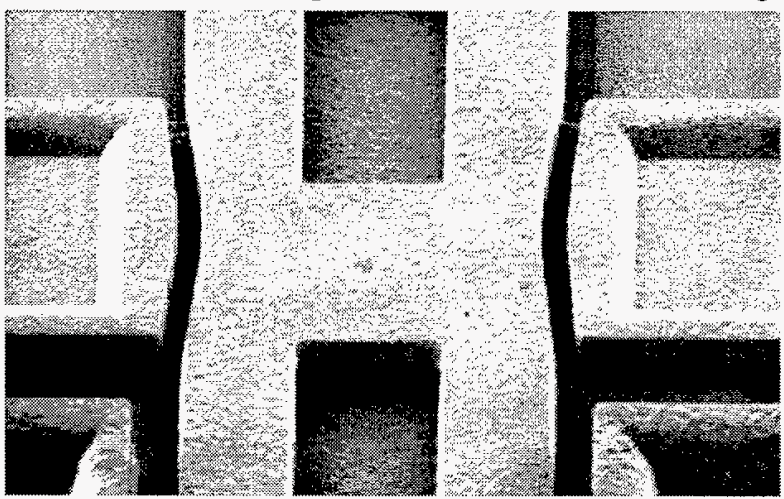

Fig. 11. Lateral clamping of a comb drive can be mitigated by design modifications such as this. When the shuttle is actuated, the gap between it and the alignment guides reduces, physically limiting the lateral displacement. 


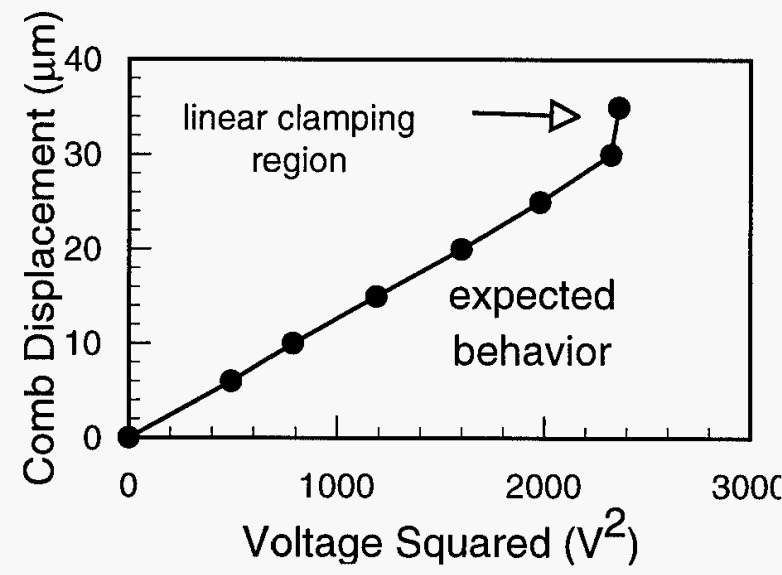

Fig. 12. Linear clamping of the comb drive results when the gap at the end of the comb fingers becomes too small, and the ideal force-voltage relationship no longer applies.

8) are constrained to remain below the buckling limit.

While the extensively used comb drive actuator was discussed as a case study in mechanical instability, similar types of instabilities can result in failure in other MEMS devices. Consequently, such issues must be considered when designing MEMS that are intended to be highly reliable.

\section{Electrical Instabilities}

The successful implementation of model-based operational methods requires a knowledge of the relationship between the applied voltage (or current) and the resulting actuation force. If this relationship deviates from that used in creating model-based drive signals, unexpected forces result that can have devastating consequences. A deviation from the expected forcevoltage relationship can result from parasitics associated with the complex 3-dimensional shape of interacting actuation electrodes. We consider once again the comb drive as a case study of how electrical instabilities can impact performance and reliability.

The static equilibrium displacement of the comb drive shown in Fig. 7, when not connected to a load, is given by combining Eqs. (1) and (2) above:

$$
y=\frac{a}{k} V^{2} \text {. }
$$

A plot of displacement versus the square of the applied voltage should result in a straight line, with slope $a / k$. This is precisely what is experimentally observed, except for large displacements. As illustrated by the data in Fig. 12, at large displacements, the position varies much more sensitively with applied voltage than expected. Equivalently, the resulting electrostatic force is larger than expected for the applied voltage. Experiments have unambiguously demonstrated that

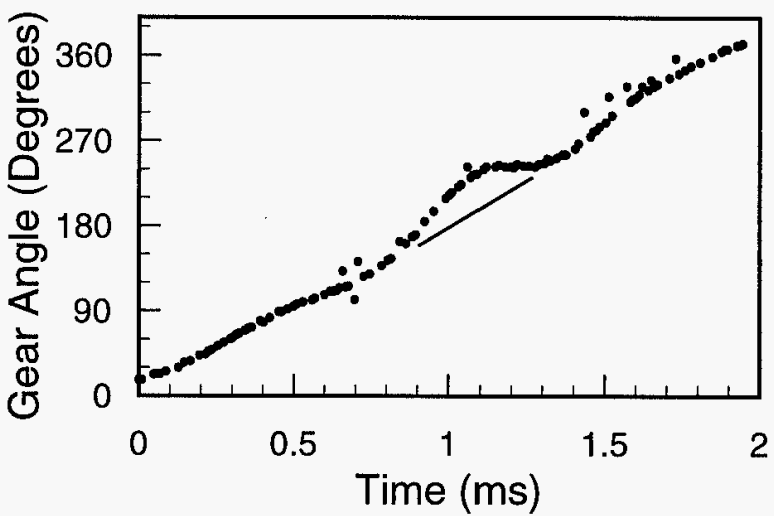

Fig. 13. Linear clamping of the comb drive causes the speed fluctuations observed at $\sim 180^{\circ}$. The pinon gear first speeds up due to the increasing electrostatic force, then briefly stops when the linear clamping occurs.

this increase in force is due to fringing fields at the ends of the comb fingers that occur when the combs are fully engaged.

The impact of this unexpected force increase on the operation of the microengine is illustrated by the data in Fig. 13. The increased force causes the pinon gear to accelerate as the comb drives become fully engaged. The gear then abruptly comes to a halt as the combs remain engaged. The electrostatic field energy favors this "linearly clamped" condition, where the system is in a local potential energy well. Motion resumes only when the applied voltage drops below the unclamping value.

As with lateral clamping, the speed fluctuations associated with linear clamping not only impact the positional accuracy of the actuation system, but result in premature degradation as well. When the electrostatic drive forces do not properly compensate the other forces in the system, constrain forces acting on rubbing or otherwise contacting surfaces result in wear and premature failure. In particular, the gear begins sticking at the $135^{\circ}$ and $270^{\circ}$ position, and eventually ceases motion at one of those two positions.

The electrical instability causing linear clamping can be mitigated by a design modification of the comb drive. Specifically, the gaps at the ends of the comb fingers when they are fully engaged are made large enough that the parasitic force due to the end fringing fields is negligible.

\section{Discussion}

Failure modes in a wide range of surface micromachined microelectromechanical actuators were examined. Interestingly, failures were not typically 
observed to be related to deficiencies in mechanical properties such as fracture strength or fatigue-related fracture. Rather, failures were typically related to contacting or rubbing surfaces. Surface-related failures occur in two categories: stiction and friction-related wear.

Stiction (the adhesion of contacting surfaces) has been demonstrated to be mitigated in several ways. Drying methods that eliminate the formation of a meniscus (Fig. 3) increase initial yield, but do not prevent stiction if subsequent contact occurs. Special chemical treatments such as coupling agents that modify the surface adhesion energy further increase yield. Finally, simply modifying the design to result in stiffer components (e.g. Fig. 4) has been shown to play a major role in increasing yield. Combining coupling agent treatments with design-related stiffening has improved yield of comb drive structures from virtually zero to nearly $100 \%$.

Friction-related wear is a dominant underlying cause of actuator failure during operation. In particular, frictional drag forces between rubbing surfaces increase during operation until they become larger than the driving forces, and motion ceases. This has been observed to occur both gradually and catastrophically. Surface treatments such as the coupling agent ODTS have been investigated, with significant reduction in friction coefficients having been observed. However, wear leading to failure still occurs.

Wear degradation is observed to be related to the forces being applied between rubbing surfaces, and lifetime can be significantly increased by reducing these forces. The primary way to reduce undesirable interfacial forces is to use model-based operational methods. Simply by modeling the system of interest, and using the model to create appropriate drive signals, lifetimes can be increased many orders of magnitude. In addition to increasing endurance, model-based operational methods significantly increase positional accuracy - an important issue for many actuation applications.

Even using model-based operational methods, the benefits can be thwarted if parasitic forces occur that are not incorporated into the model. Mechanical instabilities (e.g. buckling resulting in lateral clamping of the comb drive) or electrical instabilities (e.g. linear clamping of the comb drive) can cause forces that are difficult to incorporate into a practical, accurate operational model. These parasitic forces, when occurring in conjunction with inertial loads, can result in combined forces large enough to actually shear a pin joint. Fortunately, such instabilities and inertial effects can be mitigated by appropriate design modifications and operational methods.

\section{Summary}

Surface micromachined MEMS actuators are observed to be extremely robust regarding their mechanical properties: gear teeth do not break, joints and flexures do not break, and hubs do not fracture. Their dominant failure modes are associated with contacting or rubbing surfaces. Stiction, which limits initial yield, can be successfully mitigated by combining chemical surface treatments with mechanical design enhancements. Failures due to rubbing surfaces are accelerated by excessive parasitic forces between the surfaces. Degrading parasitic forces can be due to inertia, mechanical instabilities, electrical instabilities, and operational methods. These forces are mitigated by using model-based operational methods, and implementing mechanical design enhancements.

While illustrated using the Sandia-developed microengine, it is clear that failure modes can be mitigated and reliability significantly increased for a broad class of MEMS actuators by implementing optimized designs, operational methods, and surface treatments.

\section{Acknowledgments}

The authors are grateful to Michael Callahan and Kent Meeks for funding this work, and the personnel of the Microelectronics Development Laboratory at Sandia National Laboratories for fabricating the devices used in this study.

Sandia is a multiprogram laboratory operated by Sandia Corporation, a Lockheed Martin Company, for the United States Department of Energy under Contract DE-AC04-94AL85000.

\section{References}

1 W. Kuehnel and S. Sherman, "A surface micromachined silicon accelerometer with on-chip detection circuitry," Sensors and Actuators A, Vol. 45, No. 1, pp. 7-16 (1994).

2 R. M. Boysel, T. G. McDonald, G. A. Magel, G. C. Smith, and J. L. Leonard, "Integration of Deformable Mirror Devices with Optical Fibers and Waveguides," Proc. SPIE, Vol. 1793, pp. 34-39 (1992).

3 N. Unal and R. Wechsung, "Inkjet printheads: An example of MST market reality", Micromachine Devices, Vol. 3, No. 1, pp. 1-6, January 1998. 
4 J. J. Sniegowski, "Multi-level polysilicon surfacemicromachining technology: applications and issues", ASME 1996 International Mechanical Engineering Congress and Exposition, Proc. of the ASME Aerospace Division, November 17-22, 1996, Atlanta, GA, AD-Vol. 52, pp. 751-759.

5 More technical information regarding the SUMMiT process can be found at the web site http://www.mdl.sandia.gov/Micromachine.

6 J. J. Sniegowski, "Chemical Mechanical Polishing: Enhancing the Manufacturability of MEMS", SPIE Micromachining and Microfabrication Process Technology, Austin, TX, Oct. 14, 1996, Vol. 2879, pp. 104-115.

7 J. J. Sniegowski, S. L. Miller, G. LaVigne, M. S. Rodgers, and P. J. McWhorter, "Monolithic Geared-Mechanisms Driven by a Polysilicon Surface-Micromachined On-chip Electrostatic Engine", Technical Digest of the 1996 Solid State Sensor and Actuator Workshop, Hilton Head Island, SC, June 3-6, 1996, pp. 178-182.

8 M. S. Rodgers, J. J. Sniegowski, S. L. Miller, C. C. Barron, and P. J. McWhorter, "Advanced micromechanisms in a multi-level polysilicon technology", SPIE Micromachined Devices and Components III, Austin, TX, Sept. 29, 1997, Vol. 3224, pp. 120-130.

9 S. L. Miller, G. LaVigne, M. S. Rodgers, J. J. Sniegowski, J. P. Waters, and P. J. McWhorter, "Routes to failure in rotating MEMS devices experiencing sliding friction", Proc. SPIE Micromachined Devices and Components III, Vol. 3224, Austin, September 29, 1997, pp. 24-30.

10 H. Guckel, J. J. Sniegowski, T. R. Christenson, S. Mohney, and T. F. Kelly, "Fabrication of micromechanical devices from polysilicon films with smooth surfaces," Sensors and Actuators, Vol. 20, pp. 117-122, 1989.

11 G. T. Mulhern, D. S. Soane, and R. T. Howe, "Supercritical carbon dioxide drying of microstructures," Proceedings, International Conference on Solid-State Sensors and Actuators (Transducers '93), Yokohama, Japan, 1993, pp. 296-299.

12 R. Srinivasan, M. Houston, R. Howe, and R. Maboudian, "Self-assembled fluorocarbon films for enhanced stiction reduction", Digest of Technical Papers, 1997 International Conference on Solid-State Sensors and Actuators, Chicago, June 16-19, 1997, pp. 1399 - 1402.
13 S. L. Miller, J. J. Sniegowski, G. LaVigne, and P. J. McWhorter, "Friction in Surface Micromachined Microengines", Proc. SPIE Smart Electronics and MEMS Vol. 2722, San Diego, Feb. 28-29, 1996, pp. 197-204.

14 S. L. Miller, J. J. Sniegowski, G. LaVigne, and P. J. McWhorter, "Performance tradeoffs for a surface micromachined microengine", Proc. SPIE Micromachined Devices and Components II, Vol. 2882, Austin, October 14-15, 1996, pp. 182-191.

15 W. C. Tang, T-C. H. Nguyen, and R. T. Howe, "Laterally driven polysilicon resonant microstructures," Sensors and Actuators, Vol. 20, 1989, pp. 25-32. 
M98003046

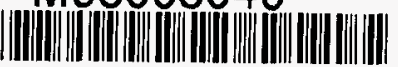
$\frac{\text { Report Number (14) SAND }-98-0382 C}{\text { CONF }-980320 \cdots}$

Jubl. Date (11) 199802

sponsor Code (18) DOE/DP, XF

JC Category (19) UC-700, DOE/ER

DOE 